\title{
SINGIBOT - A STUDENT SERVICES CHATBOT
}

\section{Aleksandar Petrović*, Miodrag Živković, Nebojša Bačanin Džakula}

Faculty of Informatics and Computing, Singidunum University,

Belgrade, Serbia

\section{Correspondence:}

Aleksandar Petrović

e-mail:

aleksandar.petrovic.17@singimail.rs

\begin{abstract}
:
Singibot is a restful web application with the intent of providing information to the students of Singidunum University. The web application takes advantage of the Spring framework for Java and the communication logic between the human and the bot is built upon the well-known AliceBot that is based on the AIML technology. The long-term goal is to build a self-learning bot that can adapt to evolving students' requests. In this iteration, we are presenting a pilot version and the implemented functions for this version are the means to respond to questions regarding the information related to the education at this institution and to carry on an informal conversation. The goal for this version is to provide a service for students that will improve their studying experience and the quality of their studies.
\end{abstract}

Keywords:

bot, communication, utility, aiml.

\section{INTRODUCTION}

The personal experience of the authors and consideration of IT trends [1] birthed the idea to produce a communications solution that can vastly improve the quality of the studies at Singidunum University. The vision is to simplify in fullness the administrative requirements for the students in terms of providing unambiguous instructions and information regarding the higher education, before admission and during the studies. The questions presented to Singibot can regard the institution, professors, length of studies, career options, working hours, tuition, academic calendar, etc. Singibot's interface consists of a chat dialog in which the user types their message in English, and after sending it they are presented with a response based on the matched pattern. It was first decided to produce an English version of the chatbot out of the priority to accommodate the requests of the foreign students. Singidunum University offers studies in Serbian and in English and due to these two official versions of the institution's website exist in these languages. To goal is to ease the burden of a novelty for the foreign and exchange students and to make their experience as fluid and as stress less as possible. 
The familiarity of semi-formal conversation paired with an impression of a well-informed person regarding the higher education facility is the core of this program which is supposed to increase the positive impact on the person from the information learned. Whilst the University's website is designed to provide the information in as simplest way as possible the situation in which an exchange student wanders around it aimlessly in search of certain classes in a particular semester of a specific study program is a hindrance even the top universities cannot pride themselves with avoiding. The complexity and the amount of information on the study programs and the classes are complicating the websites of universities. Websites of universities are required to list all information and make it available but a much better way to present information to the students is through a chatbot. With the use of an AI companion like Singibot, this information can be provided by asking for it. Due to the expensive international calls and the difficulty to speak a foreign language through phone being reserved only for the most well-versed polyglots, the situation in which a foreign student calls the facility to get information is very rare. Even if the student is currently residing in the country of studies besides the mentioned problems, the wait time on calls and the human factor are also parts of this communication that the Singibot tends to eliminate. The version of the bot in the Serbian language is part of the phase three goals, right after integrating a machine learning module.

\section{BACKGROUND AND MOTIVATION}

\section{Chatbots and AIML}

Chatbots as communication solutions are increasing in popularity but there are still some ambiguities regarding the use. The concept itself is not new but the advance of computer technologies and the situational opportunity have pushed these types of programs further. Machine learning proved to be a great combination with Artificial Intelligence Markup Language [2] invented by Richard S. Wallace. The language's data storing format provides a good foundation for building encoders and decoders to manipulate the text patterns that need to be processed with a machine learning algorithm and readable after. The stigma encompassing the use of chatbots is due to a couple of reasons. The main reason being the lack of understanding of the technology. This can be separated into two different branches on the base of the users' expertise. For the non-expert users, it is commonly the problem of comprehending the actions that would be performed by the bot, therefore this blocks the vision of the benefits that could be brought to them, similar to the problem of a non-expert user employing any other technical solution. When it comes to users with computational expertise the dilemma is if a chatbot can benefit their line of work and how much of an improvement it would contribute. The technical flaw regarding chatbots is the constant need for fine-tuning and adjusting to new data. This is a delicate process because the tweaking process can completely break the application.

\section{Similar solutions}

Higher education institutions around the globe are already deploying their chatbots or are having them in the works, independently by students and as university organized startups. The primary use of the chatbots for the university's purposes is an advanced admission procedure. The facilities already exploiting the bots pride themselves with the improved statistic of the number of active students. Student companions, as some universities call them, are psychologically analyzing the students' responses in the conversation and are providing the necessary means to the solution of the given problem. Some of them give examples of their bots at work alike when the student is failing at a certain class, the bot suggests helpful books and tutors if needed. This is possible thanks to the bot having access to students' grades to the bot. This particular case is with the bot named Beacon from Staffordshire University [3]. This is a mobile-based application with four hundred preprogrammed responses and the ability to expand to even more. It is A.I. based and supports voice and text input. The application is able to recommend activities that can positively impact students' experiences throughout their studies and send lecture reminders because it has access to each student's timetable thanks to the login process. When judged by Beacon that a student is in the need of a tutor they get informed by a message consisting of an explanation, contact information to a tutor, and an option to contact the tutor on the student's behalf forwarding the transcript of the conversation to the tutor. This and other institutions reported a decrease of students dropping out due to not being able to pass certain exams [4]. The way in which artificial intelligence is enhancing the bot is by evaluating the students' records and non-invasively offering help. Students responded much better to the suggestions of what was easily recognizable as an A.I. than to students' counselors [5]. Taking out humanity from the conversation makes students feel more comfortable opening up about their insecurities and internal struggles. 
This other university's chatbot has recognized this opportunity and took their work further. Lancaster University's chatbot named Ask L.U. [6] can talk to students about tuition, their financial situation, and similar problems. These types of conversations with the bot need to be longer so it can gather more information to act on. The idea is to develop ways to provide coaching questions and to help with anxiety management. The other functionalities of the application are timetable access, tutor recommendation, grade reviews, suggestions for finding a free computer on campus, and even an available washing machine. The bot heavily relies on Amazon's Web Services and is implemented in the university's pre-existing student companion app in the form of a voice interface. AWS CloudWatch, AWS Virtual Private Cloud, and AWS Elastic Search are the technologies that provide logging and enable the fuzzy search. Ask L.U. supports Amazon Alexa and it can be activated through it. Due to the amount of data on the students this chatbot collects it is theoretically capable of assisting students in writing essays based on their personality and previous work. Stanford University has developed a chatbot or rather QuizBot with the main purpose of helping students learn. The dialogue-based agent is designed to assist students in learning by providing an attention-grabbing interface. The app was built to compete with existing flashcard applications and the authors claim that students get $20 \%$ more correct answers with the use of QuizBot while admitting that this process is more time consuming but still taking into account that the test subjects were more engaged with the QuizBot, spending longer periods rather than using the generic flashcard app created for the study [7]. The interface of the app is based on Facebook's Messenger in which the user can have a casual conversation or answer questions by typing and clicking on a button to select one of the multiple-choice answers. There are also options for revealing the answer, asking for a hint or an explanation after the wrong answers were submitted or the student gave up. The application sends daily reminders which amongst a custom avatar and the informal conversational tone improve the user's engagement. The students' process of learning benefited from typing up answers in the same way as it would from writing them and the app showed the biggest difference in results when testing the students' knowledge after a longer period, in this case, two months. From Leeds Becket University's comes a Facebook Messenger based chatbot in the form of a desktop as well as a mobile application. Its main motivation comes from the students' extensive use of social networks for communication like WhatsApp and Facebook Messenger. The goal of the bot is to help the potential students place in the best course for them through Clearing.

\section{Benefits and impact}

Battling students dropping out, retention decrease, and troublesome mental health are the fields in which the most benefit from chatbots can be observed. Their focus is also largely on the financial situations of the students and helping them overcome it. Chatbots are becoming a pedagogical tool soon to be a crucial part of every educational facility. The most impact was detected in the admission period of the school year [8]. Communication with an A.I. medium helps students get a better insight into the coming years of their education. The result of this accomplishment is more satisfied students that are better convinced in the study program that they are choosing. Being a financially low maintenance addition to the online presence of a higher education institution chatbots can bring in the profit that justifies the cost of development. The money and energy saved on the hiring process and maintenance of the human staff that the bot is replacing are also undeniably encouraging the implementation of suchlike solutions. It is argued that the synthetic nature of the bots is actually what lets students relax and openly discuss their problems. Even though the natural assumption would be that the as more humanlike the A.I. is the more advanced it is with this scenario that is not the case. It is speculated that the impact was as big as it is because the students better responded to a service knowing that it is not human [9]. The robots that talked to them can be credited for not judging them and even ridiculing them for some trivial problems a human being would consider ridiculous. Acknowledging there are chatbots capable of consideration of serious problems that the education staff needs to be notified of, it is easier for students to confess to A.I. chatbot. The impacts and benefits to mental health should not be overlooked and in the current times, that seems to be the topic for improvement of human performance.

\section{METHODS USED AND FUNCTIONALITIES}

\section{Methods}

Singibot is a chatbot developed in the Spring framework for Java. This framework was the authors' choice for the reasons of straightforward restful development, support of dependency injection that also facilitated library integration needed to run the AIML interpreter, 
layered architecture allowing modularity, and most of all Spring's supportiveness of web applications. Singibot is a restful web application that uses STOMP (Simple Text-orientated Messaging Protocol) to send and receive messages in the chat window. The model component of the program is a class able to send and receive messages through the protocol, and it has the AIML interpreter component extracted and modified from AliceBot and it heavily relies on the $\mathrm{AB}$ library from the same program by Richard S. Wallace. The interpreter was modified in a way to accept string input extracted from a STOMP message instead of an array of strings. The class returns a string output that is written out in the chat field in the form of a response from another user. The controller is simple and contains two mappings for two types of messages the application is programmed to receive. When the user opens up the application, they are asked for the name which is stored and later accessible. This is a register type message that stores the username in its header and it activates a welcome message in a different font style than the other messages in the chat upon opening the chat. Send is the second and final type of a message the program recognizes and it is forwarding a class type that is representing a chat message as an object. This object contains the message itself and the metadata needed for the program to function properly. The front end of the application is basic HTML, CSS, and JS without using any of the popular front end frameworks which does not mean that in the future that will not be the case.

\section{Functionalities}

Every time the application is started the user is greeted with a dialog box that informs them of the action needed from them that is to enter the username and press a button to advance to the chatting window. This is the only message of register type and is initiated by pressing the button. The username is then stored and used to sign messages in the sender field which application uses to identify them. There are only two types of message senders and those are the human that has entered their username for the being of the session and the bot with the response logic behind. The welcome screen can be seen in Figure 1.

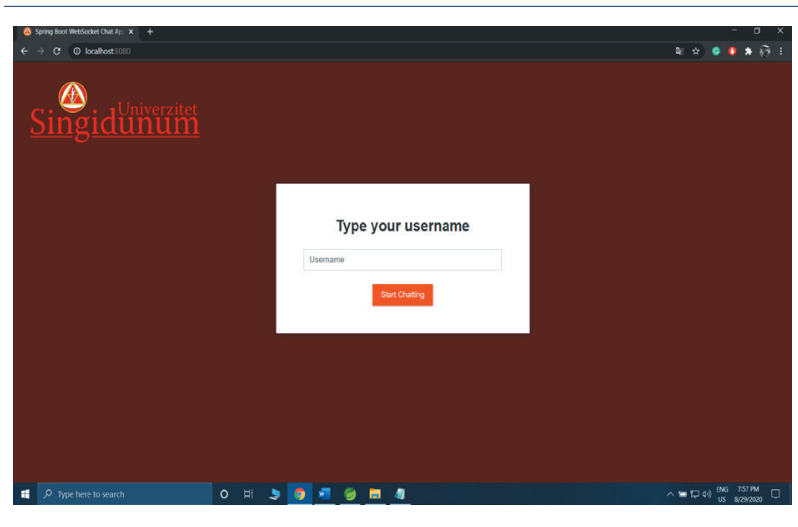

Fig. 1. Example of a welcome screen.

With every username, there is a color assigned to their avatar that is determined by the capital letter of their name. Figure 2 is depicting an example of a basic greeting and response from the bot. The color assigned for the test name "Student" and its capital letter, in this case, was a slightly different shade of red from the Singibot's color is of the Singidunum's shade of red. The bot possesses various ways of greeting and this is an example of the basic response. The function returns, in this case, the plain text which is surrounded by the conversation participant's icon and username. The importance of casual conversational abilities is paramount for the users' experience as proven from similar projects talked about in the first chapter. To rephrase, every successful solution recognized the need for an option for informal conversation, it being for relaxation periods of studying, if that was the bot's purpose, or simply humanizing the process of getting information from an artificial intelligence agent. On the contrary, the bot should not replicate human behavior too much as it decreases the effectivity of information providing due to the distractions that humans are inclined to. The user should be aware that they are talking to artificial intelligence and it should provide them with information on how it is programmed to accept and process data.

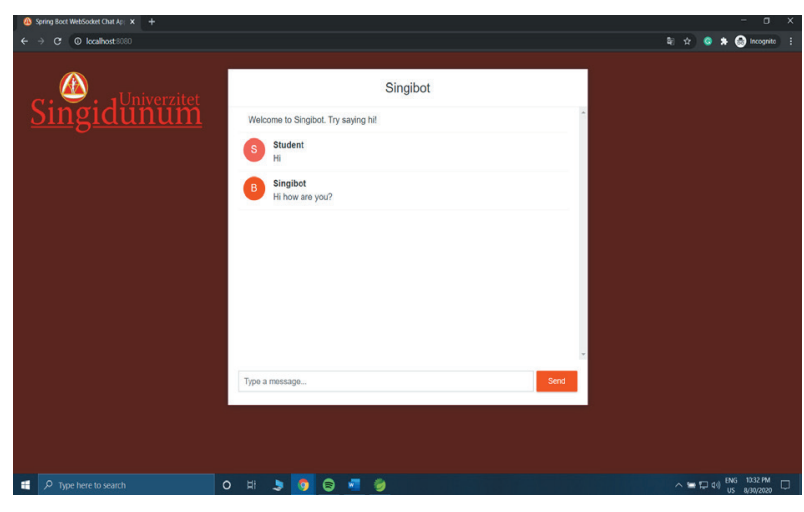

Fig. 2. Start of a conversation. 
Singibot is capable of returning live links when necessary as shown in the case in Figure 3. The link is not organically forwarded but rather later surrounded by HTML link tags through a JavaScript function. This is also an example of the robustness of Singibot's pattern recognition. The bot will respond identically to the two different user inputs that are both asking the same thing. Considering the fact there is a large number of combinations to ask the same question this program was designed to cover most of them if not all of them in some cases. The pattern recognition part will massively benefit from the implementation of machine learning as more talked about in the third chapter. The forwarded link in a different style from the chat message in this instance and clicking on it will take the user to the international Singidunum University site to the page where the professors' biographies with pictures and all of their science work can be seen. By providing this type of information students' experience is closer to the goal mentioned in the introductory chapter, familiarizing students with the teaching staff giving them better insight into what their studies will look like.

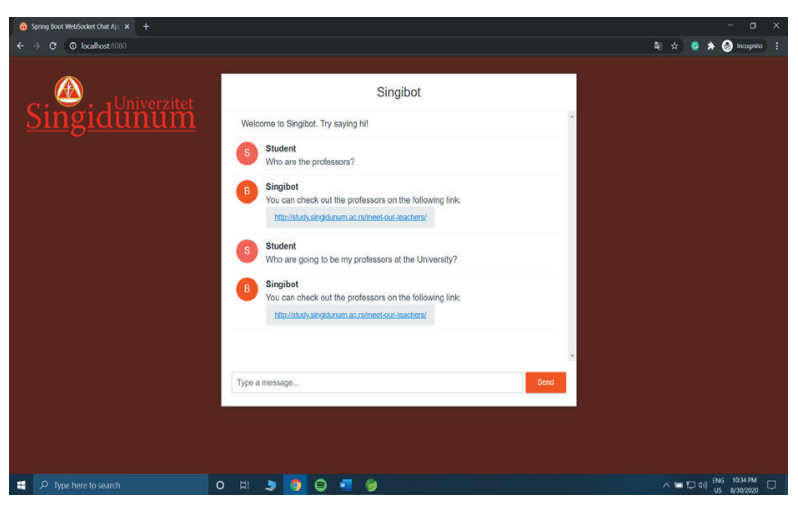

Fig. 3. Different input recognition.

Besides different student messages, the bot itself is capable of returning different responses based on a random factor that is leaving a place for improvement in phase three of the software's development. Figure 4 is depicting such behavior as the student asks in three different ways to see the website of the University and the robot responds in different ways nonrelated to the difference in asking the question but to what was the context of the question. This is further improving the user's engagement as the Singibot is heavily relying on the University's website. The main function is to provide critical information faster and easier. The questions regarding this information can be various from the number of years a study program takes to what are the working hours. Singibot can respond to these basic question and that is fundamental for the implementation of future functions. Singibot is currently capable of casual conversation and returning the most important information on the higher education facility that it was built for. The application is usable in its current state but there will be more word on the future work in the following chapter.

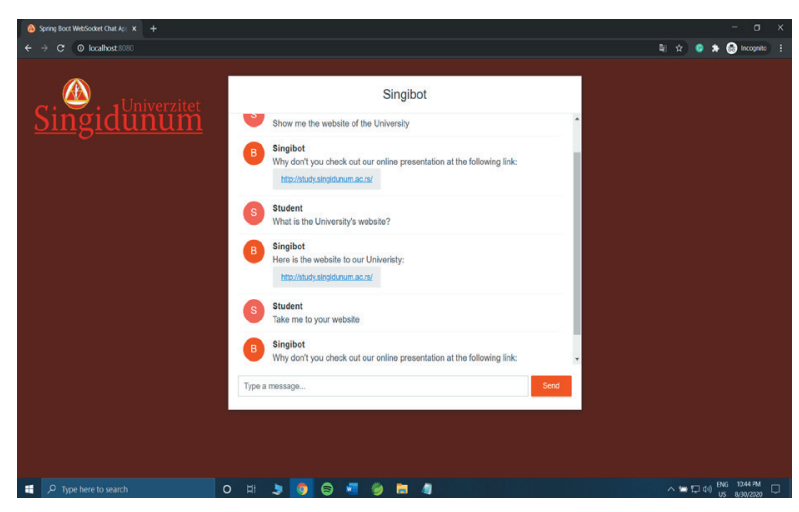

Fig. 4. Different responses and inputs.

\section{THE FUTURE}

\section{Phase three}

The currently concluding phase in the plans for the Singibot consists of implementing advanced conversational abilities, a bigger database of replies and a machine-learning algorithm to further enhance those replies and to give the program the A.I. perspective. Various solutions are being considered and even rewriting the program in Python, since its currently the leading language for machine learning, is considered an option. The chatterbots mentioned in the first chapter of this paper almost without an exception implement some sort of a learning algorithm that fortifies those creations. This is the idea the authors have for this creation also and the one presented in the paper is a pilot version to worked on in the future. It is planned to add functionality to contact a professor directly through the Singibot or put in other words to enable Singibot to contact a professor for the user. This can prove to be very useful while requesting to see a timetable of the professor's office hours to prompt the user to forward the transcript of the conversation between the student and Singibot. Professors will be getting an insight into the thought process of the student and it will make it possible for them to better understand the kind of help the student 
is in the need of. Avoidance of repeating the same conversation is also a productivity gain. Sometimes a point is not getting through an elaborate email because of the boundaries of formalities and the individuals' inability to stay within them while clearly explaining the reason for contacting a certain professor. Students should be able to contact the students' services directly through Singibot in the same manner as they will be able to contact professors. The improvements the authors believe will be observable by fulfilling the future goals for machine learning are to enhance pattern recognition and to capacitate the bot for learning new patterns and the responses to them. The effectiveness of creating a pure hardcoded response bot can be very small if not put to good use but that does not discredit the one that is currently available. Even though the limitations are obvious the Singibot is a solution for itself and it is completely usable as-is.

\section{CONCLUSION}

Singibot is a simple chatbot with a lot of space for improvement which is intended for its lifetime. The ideals of the creators are to create a support system for students that will benefit the students in the first place and after that a solution that is profitable and sustainable. Looking back on the costs and effort needed for development it is clear that the profitability equation still stands and this eliminates the excuse for not developing these types of solutions by the higher education institutions that students trust to teach the fundamentals of the matters their studying and to prepare them for a prospective career. The way the Singibot tends to improve the students' experience at this facility is through an attempt to reduce social anxiety new students experience while having to call the students' services for information. Many of the students never do that out of the said anxiety and rather stay misinformed instead for example walking into the students' services cabinet and asking those questions in person. The second most important benefit of Singibot are the results of better informed students and those are students that are successfully finishing their studies, reduced staff costs, and a better online presence of the University. Having generations that have grown up with modern technology holds some disadvantages that can be easily removed by confronting the problem in this way since the root of the problem is deeply buried in the way parents raise their kids. It was shown that the younger generations rather prefer texting than talking for which the proof is the phone com- pany operators giving more free minutes per post-paid package and more messages because most of the phone traffic is online through social communication networks and social networks. Most of the traffic the phone operators profit on is the data that is the consequence of the always-online lifestyle. These problems were all taken into consideration before the development process of the Singibot began. The idea is to make a difference no matter how small it is perceived as by the first look.

\section{REFERENCES}

[1] B. R. Ranoliya, N. Raghuwanshi and S. Singh, "Chatbot for university related FAQs," 2017 International Conference on Advances in Computing, Communications and Informatics (ICACCI), Udupi, 2017, pp. 1525-1530.

[2] R.S. Wallace, “The Anatomy of A.L.I.C.E.," Epstein R., Roberts G., Beber G. (eds) Parsing the Turing Test. Springer, Dordrecht, 2009.

[3] C. Robinson, "Impressions of viability: how current enrollment management personnel and former students perceive the implementation of a chatbot focused on student financial communication," The University of Southern Mississippi The Aquila Digital Community, higher education doctoral projects, Spring 2019.

[4] M. T. Nietzel, "How Colleges Are Using Chatbots To Improve Student Retention,” Forbes, March 2020.

[5] R. Winkler, M. Söllner, "Unleashing the Potential of Chatbots in Education: A State-Of-The-Art Analysis," Academy of Management Annual Meeting (AOM) Chicago, USA., 2018.

[6] A. Mari, "Lancaster University enhances student engagement with cloud-based chatbot, Computer Weekly, blog, 12. April 2019. 